\title{
Hysteresis losses in hollow superconducting filaments and in multifilament systems
}

\author{
J.A. Eikelboom and L.J.M. van de Klundert* \\ Netherlands Energy Research Foundation ECN, PO Box 1, 1755 ZG Petten, \\ The Netherlands \\ *University of Twente, PO Box 217, 7500 AE Enschede, The Netherlands
}

Received 13 December 1990

\begin{abstract}
The magnetization of hollow superconducting filaments in a perpendicular applied field is calculated numerically using a mathematical model. With this model the hysteresis losses over a closed cycle of the applied field, as well as the instantaneous power dissipation in the filament, can be calculated. Theoretical results are compared with data from experiments using wire with hollow $\mathrm{Nb}_{3} \mathrm{Sn}$ filaments. Demagnetization effects caused by the influence of the field of one filament on another are studied and, finally, bridging between neighouring filaments and its influence on the effective filament diameter and the hysteresis losses are discussed.
\end{abstract}

Keywords: superconductors; hysteresis losses; hollow filaments; demagnetization; effective filament diameter

Hysteresis losses in solid circular filaments are well understood ' and expressions based on the relation between the calculated magnetization and the applied magnetic field have been proposed by several authors (see, for example, Reference 2). Several numerical techniques were used by these authors to determine the location of the moving boundary between penetrated and non-penetrated regions in the filament. Knowing this location, it is possible to calculate the resulting magnetization and the hysteresis losses for a closed cycle of the applied field. The results of these different approaches showed good agreement. All the techniques used, however, lacked the capability to treat more complicated situations, e.g. losses in hollow filaments, filaments with transport currents or situations where the critical current density depends on the magnetic field.

The mathematical model developed by Hartmann ${ }^{3}$ has the advantage of being based only on the fundamental Maxwell equations and the constitutive equations given by the Bean model. The model allows the critical current density to be dependent on the local magnetic field. The magnetization of filaments with arbitrary cross-section can be calculated in cases with and without transport current. Adjoining filaments are coupled by their induced magnetic fields. The influence of these demagnetization effects on the current distribution can be studied. Bridging between neighbouring filaments has disastrous effects on the hysteresis losses. The increased magnetization as a function of the applied field can be calculated for models of bridged filaments.

Usually expressions for hysteresis losses over a closed cycle of the external field are given. The fact that the cycle is closed guarantees that the current distribution at the beginning is the same as that at the end of the cycle. This means that the energy stored in the magnetic field produced by the current distribution need not be taken into account. Changes in the stored energy play a significant role in the power balance of the filament. In general the instantaneous power dissipation is not equal to the work performed by the external field at the same moment. Both the energy stored in the current distribution as well as the instantaneous power dissipation can be calculated using the model.

\section{Mathematical model}

Mathematical details of the model used here have been extensively dealt with by Hartmann ${ }^{3}$. Only a brief outline of the characteristics will be given here. Consider an infinitely long superconductor in an external perpendicular magnetic field where all the physical characteristics, such as fields and dimensions, are invariant with respect to translations along the axis. It is assumed that there is no transport current. Changes in the external field will induce an electric field parallel to the axis and this field will drive the local current density in the superconductor to its critical value. The basic laws which govern this phenomenon are the Maxwell equations, together with the constitutive equations representing the Bean model

$$
\left|j_{z}\right| \leq j_{\mathrm{c}} \text { if } E_{z}=0 \text {, otherwise } j_{c} \sin \left(E_{z}\right)
$$


These relations determine the current distribution inside the superconductor. The mathematical model is based on an expression for the vector-potential $\vec{A}=\left(0,0, A_{z}\right)$ which is derived from the Maxwell equations. The $z$ component of $\vec{A}$ is given by

$$
\begin{aligned}
A_{z}(\vec{r}, t)= & -\frac{\mu_{0}}{2 \pi} \int_{S} \log \left|\vec{r}-\overrightarrow{r^{\prime}}\right|_{j_{z}}\left(\partial_{\mathrm{t}} A_{z}\right) \mathrm{d} \vec{r} \\
& -\left[\vec{r} \times \overrightarrow{B_{\mathrm{a}}}(t)\right]_{z}+C(t)
\end{aligned}
$$

$C(t)$ is determined by the transport current, $I_{\mathrm{tr}}$, in the filament; $C(t)=0$ for $I_{\mathrm{tr}}=0$.

This expression is useful because the electric field, $\vec{E}=\left(0,0, E_{z}\right)$, which determines the current distributions, is given by

$$
E_{z}(\vec{r}, t)=-\partial_{1} A_{z}(\vec{r}, t)
$$

With a certain current distribution inside a superconductor a change in the applied field may lead to a change in the local vector-potential; this depends on the current density changes elsewhere in the superconductors. The local appearance of an electric field in its turn may lead to an altered current density; this depends on the existing current density and is governed by the constitutive equations. The well-known fact that the induced currents tend to screen the interior parts of the superconductor from the external field change is a consequence of fundamental laws and is assumed nowhere in the present model.

The field experienced by local currents is obviously not the external field but the sum of the external field and the field originating from induced currents in other parts of the superconductor. The local critical current density is determined by this field. In the present model a relation between the critical current density and the field, e.g. the Kim relation, can be assumed. It is also possible to treat the case where there is a transport current in the superconductor, but this is beyond the scope of this article.

The governing equations can be solved numerically using a time-stepping process, where the external field change is divided into a number of field steps, $d B$. The cross-section of the superconductor is approximated by a set of a large number of square cells with centres $r_{k}$. The mean current density, $j_{m}$, can be defined for every cross-section, $S_{k}$. For this set of cells the integral in the expression for the vector-potential $\vec{A}$ can be discretized for $A_{z}$ at $r_{k}$. In the numerical model the current distribution at the beginning of a field step $d B$ is given and the distribution at the end of the field step is required. If a certain end distribution is to be tested for correctness, the discretized expression for the vector-potential yields a number of equations from which the change in the vector-potential, $A\left(\vec{r}_{\mathrm{k}}\right)$, can be calculated. This change is proportional to the electric field and it can be checked whether the proposed distribution satisfies the constitutive equations. If this is not the case, the distribution is changed and checked again until a correct distribution is found.

In a typical situation the cross-section of a solid circular filament is approximated by a set of several hundred cells, and the field steps are typically $1 / 50$ of the penetration field. For most field steps a few iteractions are sufficient to find the current distribution. From the current distributions the magnetization of the superconductor is easily obtained and from calculated variables such as vector-potential and electric field other physical quantities such as stored energy and dissipated energy can be calculated.

\section{Hollow filaments}

Several aspects of losses in hollow filaments have been considered in previous articles ${ }^{4,5}$. The penetration of a solid circular filament of radius $R$ has been calculated by several authors, all of whom used the fact that the boundary between saturated and non-saturated regions in the filament moves in such a way so as to shield the interior region of the filament from the field change. When the applied field, $B_{\mathrm{a}}$, increases from $B_{\mathrm{a}}=0$ in an initially current-free filament, the centre of the filament is the last point to be reached by the boundary when $B_{\mathrm{a}}=B_{\mathrm{p}}$, the penetration field of the filament

$B_{\mathrm{p}}=\stackrel{2}{\pi} \mu_{0} j_{\mathrm{c}} R$

With $\beta=B_{\mathrm{a}} / B_{\mathrm{p}}$ the following relation between the magnetization and the applied field is found as a fit to numerical results (see Reference 3 )

$M=\left[\frac{2}{3}\left[(1-\beta)^{3}-1\right]+0.16(1-\beta)^{2} \beta^{2}\right] \frac{B_{\mathrm{p}}}{\mu_{0}}$

The magnetization of a fully penetrated filament is thus given by

$M_{\mathrm{sol}}=\frac{2}{3} \frac{B_{\mathrm{p}}}{\mu_{0}}$

The magnetization of an initially current-free hollow filament having outer radius $R_{2}$ and inner radius $R_{1}$ is equal to that of a solid filament as long as the applied field, $B_{\mathrm{a}}$, is smaller than $B_{\mathrm{p} 1} ; B_{\mathrm{p} 1}$ being the field at which the moving boundary reaches the inner radius, $R_{1}$. We define $B_{\mathrm{p} 2}$ as being the field at which the hollow filament is totally penetrated. It is clear that the penetration field, $B_{\mathrm{p} 2}$, and the saturated magnetization, $M_{\mathrm{sat}}$, of a hollow filament will be smaller than those of a solid filament with the same outer radius. The value of the saturated magnetization of a hollow filament can be easily calculated to be

$M_{\mathrm{hol}}=\left[1-\rho^{3}\right] M_{\mathrm{sol}}, \quad$ with $\quad \rho=R_{1} / R_{2}$

The penetration field of a filament is equal to the highest field induced by the current distribution in a saturated filament. In a solid filament the current distribution produces a field $B_{\mathrm{p}}$ in the centre and this is the last point where the external field can be cancelled. In a hollow filament the highest induced field is $B_{\mathrm{p} 2}$ and it is located at two points on the symmetry axis dividing the current distributions of opposite sign, between $R_{1}$ and $R_{2}$ on 
both sides of the central hole. When the current density is constant one can calculate the induced field analytically using the Biot and Savart law and locate the maximum. When the external field is between $B_{p 1}$ and $B_{\mathrm{p} 2}$, the boundary still moves in such a way as to screen interior regions of the superconductor. If $B_{\mathrm{a}}$ increases beyond $B_{\mathrm{p} 1}$, the boundary closes so as to remain inside the superconductor. On both sides of the hole islands of non-penetrated superconductor are formed, which shrink in size as $B_{\mathrm{a}}$ increases towards $B_{\mathrm{p} 2}$. The last point to be reached is the point where the field induced by the screening currents is $B_{\mathrm{p} 2}$.

Figure 1 shows the contours for a hollow filament with $\rho=0.6$, where the field steps are $0.05 B_{\mathrm{p}}$. It can be seen that $0.30 B_{\mathrm{p}}<B_{\mathrm{pl}}<0.35 B_{\mathrm{p}}$ and $0.60 B_{\mathrm{p}}<$ $B_{\mathrm{p} 2}<0.65 B_{\mathrm{p}}$. The contours shown are fits to the boundaries found. The value of $B_{\mathrm{p} 1}$ can only be calculated using the numerical model, while $B_{\mathrm{p} 2}$ can be determined from the maximum field found. Good approximations are determined to be

$B_{\mathrm{p} 1}=\left(1.000-1.395 \rho+0.579 \rho^{2}-0.184 \rho^{3}\right) B_{\mathrm{p}}$

$B_{\mathrm{p} 2}=\left(1.000-0.331 \rho-0.074 \rho^{2}-0.596 \rho^{3}\right) B_{\mathrm{p}}$

Figure 2 shows $\beta_{1}=B_{\mathrm{p} 1} / B_{\mathrm{p}}$ and $\beta_{2}=B_{\mathrm{p} 2} / B_{\mathrm{p}}$ as a function of $\rho$, the ratio between the inner radius, $R_{1}$, and outer radius, $R_{2}$.

The calculated magnetization of a solid filament and a hollow filament are shown in Figure 3 for several values of $\rho$. For a hollow filament three regions can be distinguished: $1, B_{\mathrm{a}} \leq B_{\mathrm{pl}} ; 2, B_{\mathrm{p} 1} \leq B_{\mathrm{a}} \leq B_{\mathrm{p} 2}$; and 3 , $B_{\mathrm{p} 2}<0.65 B_{\mathrm{p}}$. The contours shown are fits to the boundaries found. The value of $B_{\mathrm{p} 1}$ can only be calculated using the numerical model, while $B_{\mathrm{p} 2}$ can be determined from the maximum field found. Good approximations are determined to be

$M_{2}(\beta)=M_{\mathrm{hol}}+\frac{a B_{\mathrm{p}}}{\mu_{0}}\left(\beta_{2}-\beta\right)^{n}$

Expressions for the coefficients $a$ and $n$ are found by taking into account the requirement that the magnetiza-

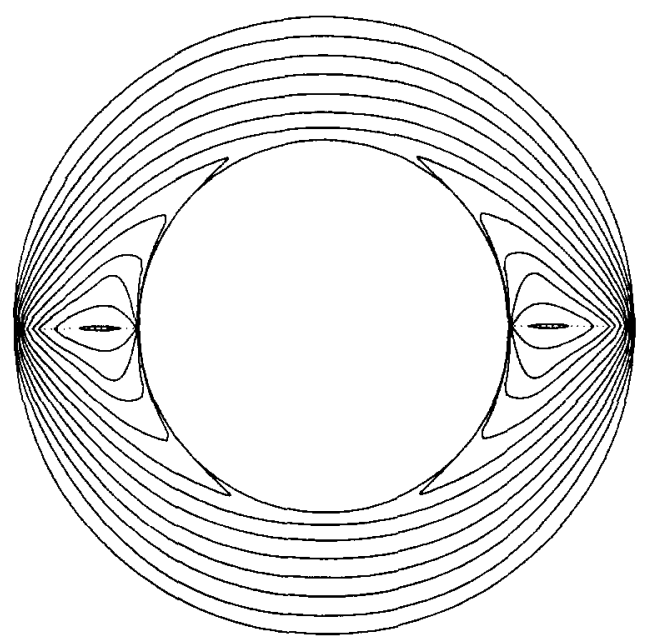

Figure 1 Contours of current penetration in a hollow filament with $\rho=0.6$. The field step is $0.05 B_{\mathrm{p}}$

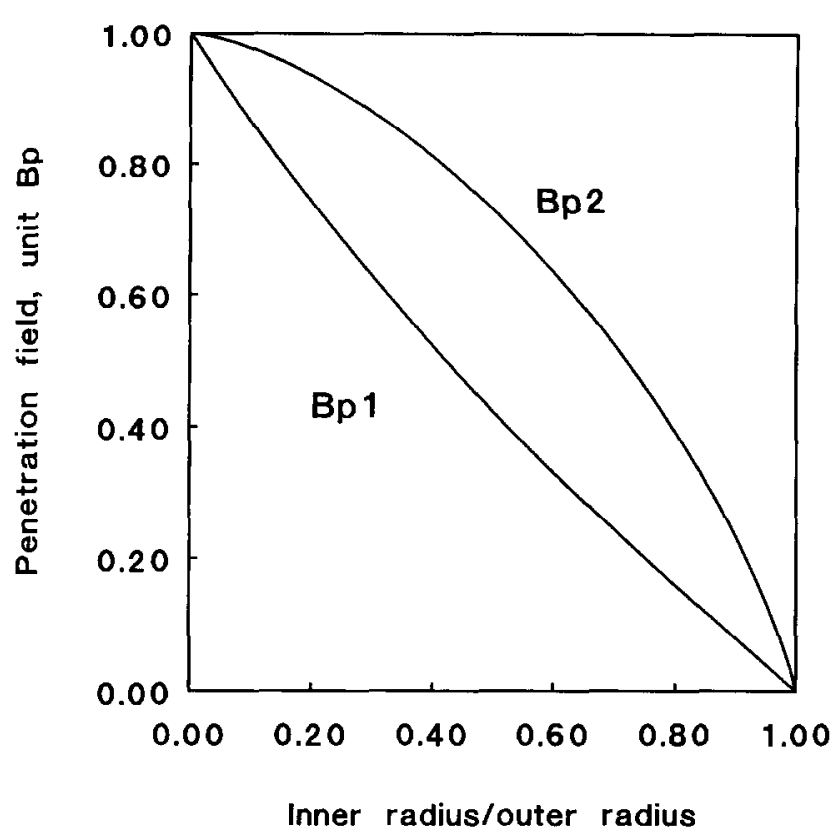

Figure 2 Penetration fields $B_{\mathrm{p} 1}$ and $B_{\mathrm{p} 2}$ scaled to $B_{\mathrm{p}}$ as a function of $\rho=R_{1} / R_{2}$

tion and its derivative are continuous at $\beta=\beta_{1}$. In Figure 3 region 2 begins at the field where the magnetization for a certain value of $\rho$ becomes less than that of a solid filament and the boundary between region 2 and region 3 is indicated by a dashed line.

Hysteresis losses, $Q$, per unit volume per cycle can be calculated from the expressions for the magnetization (see the formulae in a previous publication on this subject ${ }^{4}$ ). In order to compare losses in hollow filaments with losses in solid ones it is useful to define the loss factor, $\Gamma(\delta)$, as follows

$\Gamma(\delta)=Q(\delta) \frac{2 \mu_{0}}{\left(B_{\mathrm{p}}\right)^{2}}$

where $\delta=\Delta B / B_{\mathrm{p}}$, with $\Delta B$ being the field variation during one field cycle. $\Gamma(\delta)$ is shown in Figure 4 for

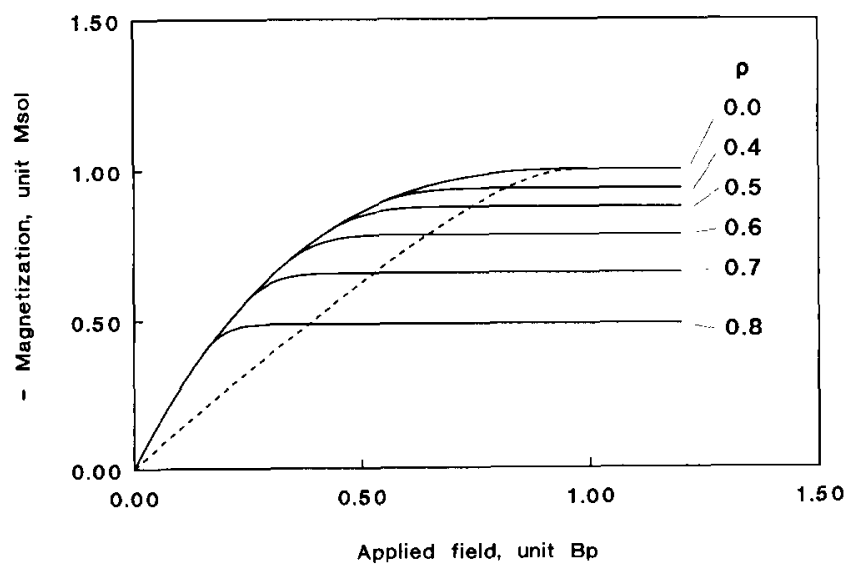

Figure 3 Magnetization scaled to $M_{\text {sol }}$ of a solid filament and a hollow filament with $\rho=0.0,0.4,0.5,0.6,0.7$ and 0.8 versus applied field, $B_{\mathrm{a}}$, scaled to $B_{\mathrm{p}}$. The dashed line connects the points where the filaments are totally penetrated 


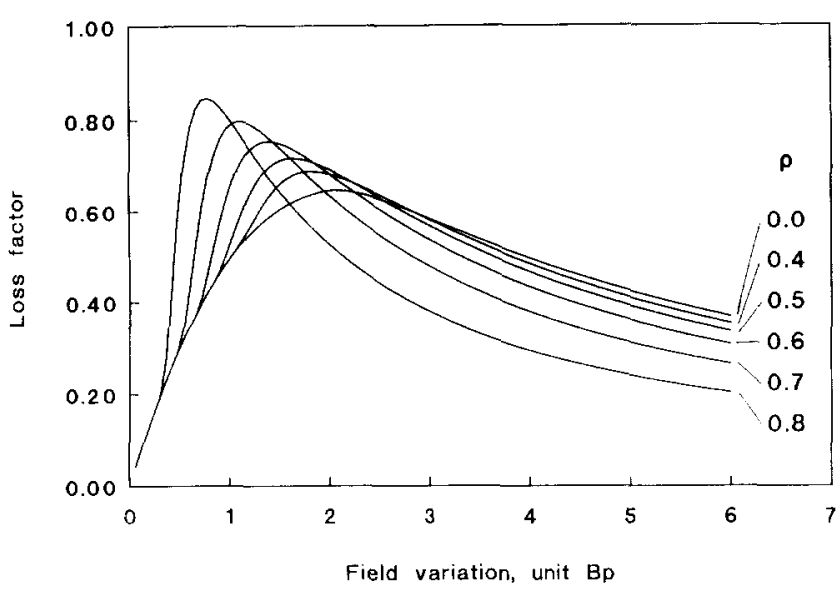

Figure 4 Loss factor, $\Gamma(\delta)$, as a function of $\rho$, with $\rho=0.0,0.4$ $0.5,0.6,0.7$ and 0.8

several values of $\rho$. It is seen that for $2 \beta_{1}<\delta<2 \beta_{2}$, losses in hollow filaments are higher than in solid filaments having the same outer diameter, whereas when $\delta$ is sufficiently greater than $2 \beta_{2}$, they are smaller. Compared to a solid filament with the same superconducting area the losses in a hollow filament will be higher for $\delta \gg 2 \beta_{2}$. The slope of the curves increases discontinuously at $\delta=2 \beta_{1}$, because of the discontinuity in the derivatives of the magnetization curve at this point, whereby the area of the magnetization loop is increased.

Figure 5 shows the hysteresis losses measured in superconducting wire with 36 hollow $\mathrm{Nb}_{3} \mathrm{Sn}$ filaments. The wires were produced by a powder method developed at $\mathrm{ECN}^{6}$. The magnetization was measured by balanced induction coils and the losses were determined calorimetrically. In the figure a measurement at a bias field of $2.0 \mathrm{~T}$ and a pulse field, $\Delta B$, of $0.6 \mathrm{~T}$ corresponds to the loop $2.0 \rightarrow 2.6 \rightarrow 2.0 \mathrm{~T}$. A curve for a certain pulse field can be compared with the curves of Figure 4. Here $\delta$ increases with the bias field because the penetration field decreases. In the experimental data the ditch at $\delta=2 \beta_{1}$ can be seen in the curves for $\Delta B=0.4$

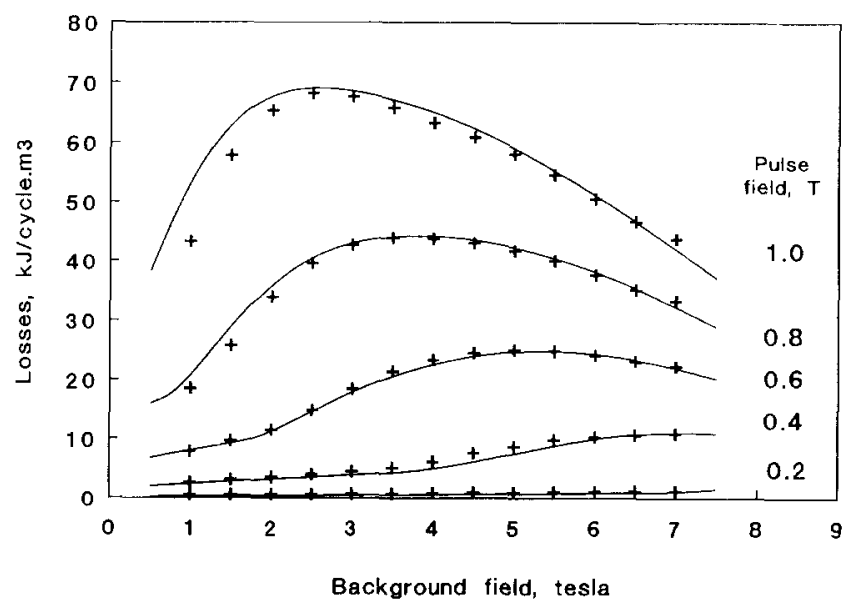

Figure 5 Calculated and measured losses in wire with hollow filaments, at pulse field strengths $0.2,0.4,0.6,0.8$ and $1.0 \mathrm{~T}$ and background fields between 0.5 and $7.5 \mathrm{~T}$ and $0.6 \mathrm{~T}$. For $\Delta B=0.8 \mathrm{~T}, \delta>2 \beta_{2}$ and for $\Delta B=0.2 \mathrm{~T}, \delta<2 \beta_{1}$.

The hysteresis losses, $Q$, per cycle were calculated by means of expressions which assume a constant critical current density. The mean field $B+\Delta B / 2$ was taken as the field which determines the effective critical current density. For small background fields and large pulse fields, $\Delta B$, this will give rise to relatively large errors which otherwise are known to be small. The $j_{\mathrm{c}}(B)$ relation was derived from a fit to the measured losses by means of the developed theory and was given by the Kim relation $j_{c}(B)=j_{c}(0) B_{0} /\left(|B|+B_{0}\right)$, with $j_{c}(0)=8.66 \times$ $10^{10} \mathrm{~A} \mathrm{~m}^{-2}$ and $B_{0}=1.51 \mathrm{~T}$ for $B<4.0 \mathrm{~T}$, and by a polynome $j_{c}(B)=\left(4.36-0.56 B+0.016 B^{2}\right) \times 10^{10} \mathrm{~A}$ $\mathrm{m}$ ? for $B>4.0 \mathrm{~T}$. In the fit. $R_{1}=27 \mu \mathrm{m}$ and $R_{2}=39 \mu \mathrm{m} . \quad R_{1}$ and $R_{2}$ are as determined from photographs of the cross-section of the filaments. The function $j_{i}(B)$ may be considered a free parameter. The fact that a good fit is found over the whole range of bias and pulse fields with corresponding ditches in both experimental and theoretical data is an indication of the reliability of the model proposed.

\section{Power balance}

Parts of the theoretical formalism of the power balance in a superconducting filament have been dealt with in a previous publication ${ }^{5}$. A changing magnetic field causes an electric field in a superconductor. The electric field and the resulting current give rise to energy dissipation in the form of heat. The power density, $P_{\mathrm{H}}$, generated is given by

$P_{13}=\left.\frac{1}{S}\right|_{S} E_{-}(\vec{r}, t) j_{-}(\vec{r}, t) \mathrm{d} \vec{r}$

The only agent that can perform work is the external magnetic field, the work being equal to

$P_{\mathrm{B}}=-\vec{M} \cdot \partial_{\mathrm{t}} \overrightarrow{B_{\mathrm{a}}}$

The work, $P_{\mathrm{B}}$, performed, however, is not always equal to the dissipated heat, $P_{\mathrm{H}}$. As $M$ and $\partial_{1} B$ can have the same sign (immediately after field reversal), $P_{\mathrm{B}}$ can even be negative, whereas $P_{\mathrm{H}}$ should always be positive as $E$, and $J$, have the same sign. The missing energy is the amount stored as magnetic energy in the current distribution. In the same way as energy is stored in an inductive c rcuit carrying a current, energy can be stored in or taken out of the current distribution in a filament. Changes in the magnetic energy density are given by

$\mathrm{d} \epsilon_{\mathrm{M}}=\left.\frac{1}{S}\right|_{S} j_{z} \cdot \frac{\partial A_{z}^{\mathrm{ind}}}{\partial t} \mathrm{~d} \vec{r}$

where $\vec{A}^{\text {ind }}$ is the vector-potential induced by the current distribution. The power balance can be formulated mathematically if we subsititute Equation (3) in Equa- 
tion (12), which gives

$$
\begin{aligned}
P_{\mathrm{H}}= & \frac{\mu_{0}}{2 \pi S} \int_{S} j_{z}(\vec{r}, t) \partial_{\mathrm{t}}\left[\int_{S} \log \left|\vec{r}-\overrightarrow{r^{\prime}}\right|_{z}(\vec{r}, t) \mathrm{d} \vec{r}\right] \mathrm{d} \vec{r} \\
& +\frac{1}{S} \int_{S} j_{z}(\vec{r}, t)\left(\vec{r} \times \partial_{\mathrm{t}} \overrightarrow{B_{\mathrm{a}}}\right)_{z} \mathrm{~d} \vec{r} \\
& -\frac{1}{S} \int_{S} j_{z}(\vec{r}, t) \frac{\mathrm{d} C(t)}{\mathrm{d} t} \mathrm{~d} \vec{r}
\end{aligned}
$$

The first term is similar to Equation (14) and we can define the power density, $P_{\mathrm{M}}$, stored as magnetic energy as

$P_{\mathrm{M}}=\frac{-\mu_{0}}{2 \pi S} \int_{S} j_{z}(\vec{r}, t) \partial_{\mathrm{t}}\left(\int_{S} \log \left|\vec{r}-\vec{r}^{\prime}\right| j_{z}\left(\vec{r}^{\prime}, t\right) \mathrm{d} \vec{r}^{\prime}\right) \mathrm{d} \vec{r}$

The second term in Equation (15) can be seen to be equal to Equation (13). As the constant, $C(t)=0$ for the case of a filament without transport current, the third term is zero. The dissipated heat can come from two sources as the two terms in this equation indicate: from stored energy or from the external field. We can write the power balance as

$P_{\mathrm{H}}=-P_{\mathrm{M}}+P_{\mathrm{B}}$

which can be drawn schematically as follows

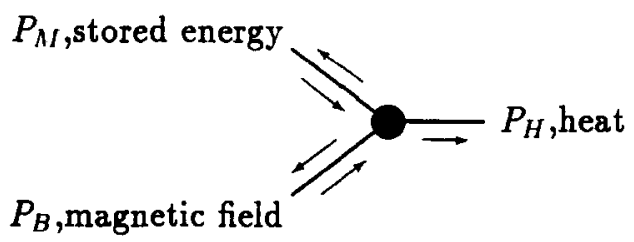

Figure $6 a$ shows the applied field for a triangular field variation as a function of the field sweep modulus, $B^{*}$, which we define as $B^{*}=\Sigma\left|B_{\mathrm{i}}\right|$. As 'time', is not a variable in the model, the field sweep modulus as defined above is used instead because, as $\left|\partial_{\mathrm{t}} \vec{B}_{\mathrm{a}}\right|=$ constant for triangular field sweeps, it is seen that $\Sigma\left|B_{\mathrm{i}}\right| \propto$ time.

Figures $6 b-d$ show the components $P_{\mathrm{B}}, P_{\mathrm{M}}$ and $P_{\mathrm{H}}$ of the power balance, as they can be calculated with the numerical model. On the horizontal axis the field sweep modulus is plotted and on the vertical axis the converted energy per unit field change (in units of $B_{\mathrm{p}}$ ). The filament is solid with an outer radius of $1(\mathrm{~m})$. The local critical current density, $j_{\mathrm{c}}(\vec{r})$, is determined by the local magnetic field, $B(\vec{r}), j_{\mathrm{c}}=1 /(1+|B|)\left(\mathrm{A} \mathrm{m}^{-2}\right)$. For triangular field sweeps, the converted energy is proportional to the power converted instantaneously.

It is seen that the external field performs negative work immediately after a field sweep reversal. This energy originates from the stored magnetic energy in the current distribution, which changes rapidily, whereas the dissipated heat is almost negligible. The heat dissipation peaks at the end of both the up-ramp and the downramp, being $\approx 17 \%$ greater during the down-ramp.
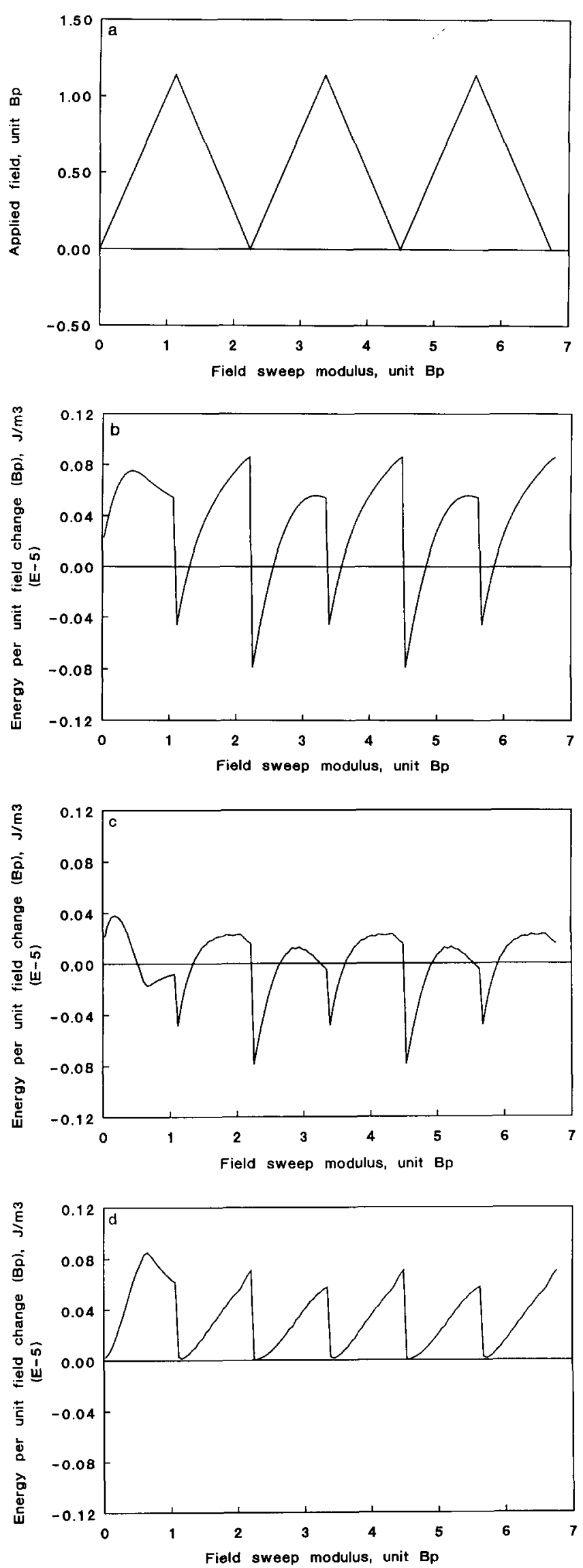

Figure 6 Applied field and calculated power balance for a wire with perpendicular field sweep $0 \rightarrow 1.14 \rightarrow O B_{p}$ repeated three times, with $j_{c}$ determined by $|B|$. (a) Applied field for a triangular field variation as a function of field sweep modulus; (b), (c), (d) components $P, P_{\mathrm{M}}$ and $P_{\mathrm{H}}$ of the power balance, respectively, as calculated with the numerical model 
Figure $7 a$ shows the calculated magnetization as a function of applied field for the cycles of Figure 6. The filament is initially current-free and the calculated magnetization is shown for the sweep $0 \rightarrow 1.14 \rightarrow 0 \rightarrow$ $1.14 \rightarrow 0 B_{\mathrm{p}}$. The second sweep, $0 \rightarrow 1.14 B_{\mathrm{p}} \rightarrow 0 B_{\mathrm{p}}$, is different from the first one because of the persistent currents which remain in the filament after the first sweep.

Figure $7 b$ shows the measured magnetization loop from an experiment where a sample of superconducting wire was located in a perpendicular field which made triangular field sweeps between 0 and $1 \mathrm{~T}$ with a period of $44 \mathrm{~s}$. The magnetization is scaled to a maximum of 100. The loop is quite asymmetrical because of the large penetration field of the filaments at low fields. The curves of Figure $7 a$ and $b$ are seen to have almost identical shapes.

The superconducting wire was produced by Teledyne Wah Chang Albany Company by the modified jelly roll
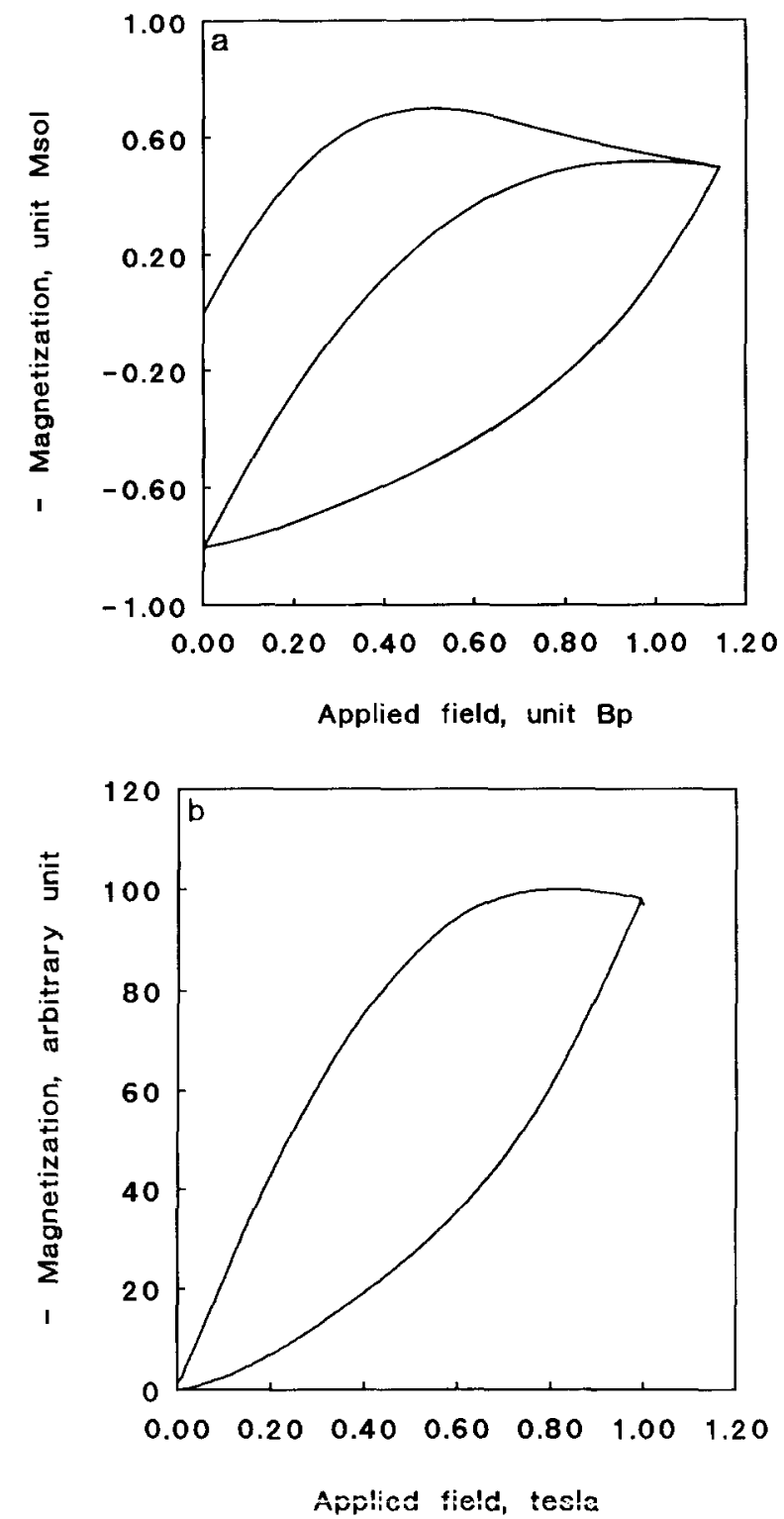

Figure 7 (a) Calculated magnetization loop for the cycle in Figure 6 with magnetization scaled to 100 ; (b) measured magnetization loop in superconducting wire experiencing a perpendicular field with cycle $\mathrm{O} \rightarrow 1 \rightarrow \mathrm{OT}$ method and had many thin $\mathrm{Nb}_{3} \mathrm{Sn}$ filaments. The effective filament diameter for this sample is know to be rather large because of bridging between the filaments. When hysteresis lossses are measured calorimetrically by the boil-off technique, the measured flow of helium gas is an indication of the dissipated heat, $P_{\mathrm{H}}$.

Figure 8 shows the gas flow signal over several minutes from the experiment of Figure $7 b$. The gas flow is seen to have the same period of $44 \mathrm{~s}$ as the pulsating field, with two maxima per cycle, one somewhat higher than the other. When the field variation was stopped at $0 \mathrm{~T}$, the last peak was the higher one, which therefore corresponds to the ramp down from 1 to $0 \mathrm{~T}$. This difference corresponds qualitatively with the calculated heat dissipation, $P_{\mathrm{H}}$, of Figure $6 d$. The fact that the gas flow signal in Figure 8 does not come down to zero after a change in field sweep direction and differs in shape from Figure $6 d$ is due to the fact that the volume of the tubes and pipes between the cryostat and the flowmeter in the experiment behaves like a buffer, whereby the signal is filtered.

\section{Multifilament systems}

\section{Demagnetization effects}

In a wire or a cable a superconducting filament is surrounded by a large number of other filaments. Although the magnetic field generated by the current distribution of a saturated filament decreases rapidly outside the filament, its effects on the current distribution in neighbouring filaments are measurable. $\operatorname{Rem}^{7}$ calculated the influence of the demagnetization factor on the hysteresis losses under the assumption that the field in a filament induced by neighbouring filaments is uniform. This assumption does not have to be made in the present model, which can also treat cases where the filaments almost touch each other. To estimate the size of demagnetization effects, nine solid circular filaments are put in a row at distances, $l$, between them of $l=0.1$, $0.3,0.6,1.0$ and 2.0 times the radius of the filament. If this configuration is positioned in an increasing magnetic field, the resulting magnetization depends on

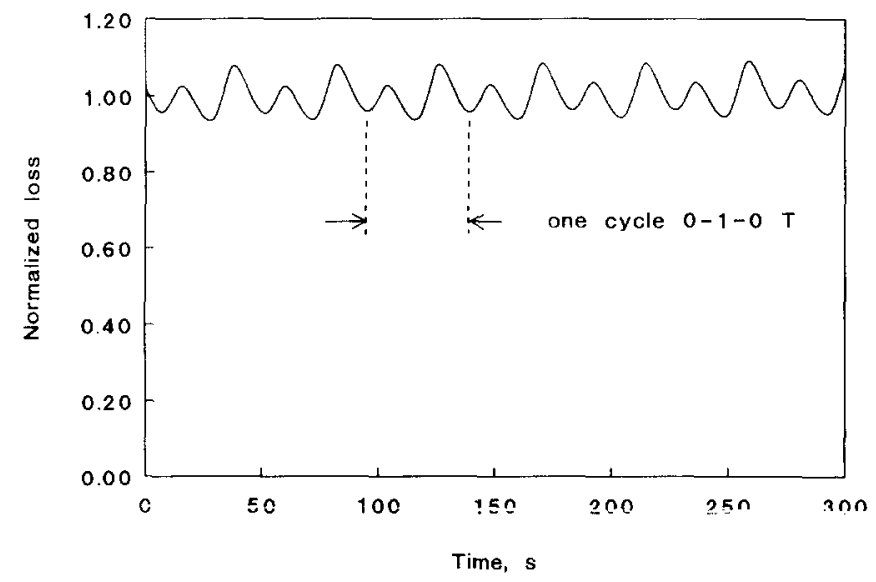

Figure 8 Signal from measured gas flow from a superconducting wire experiencing a perpendicular field with cycle $\mathrm{O} \rightarrow 1 \rightarrow \mathrm{OT}$ 
whether the applied field is alongside the broad face of the row or perpendicular to it.
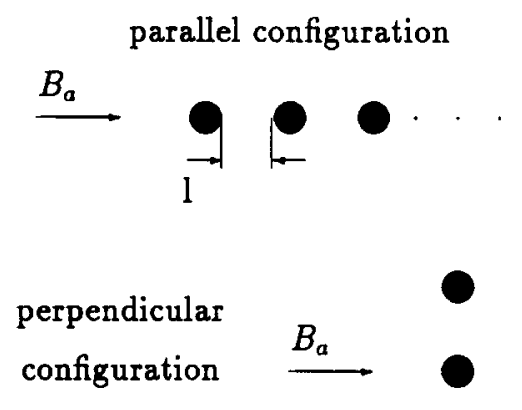

When the applied field is parallel to the broad face, the current distribution induced in one filament generates a magnetic field which at the site of neighbouring filaments is opposite to the applied field. So the effective field experienced by each filament is somewhat smaller than the applied field and the magnetization increases more slowly than in a monofilament. On the other hand, when the applied field is perpendicular to the broad face the situation is reversed, each filament experiencing a field greater than the applied field. In this case the magnetization increases more rapidly than in a monofilament.

Figure 9 shows the magnetization of a row of nine circular filaments in the perpendicular and the parallel field configuration. In the centre is the magnetization of a monofilament which is equal to that of a configuration of filaments with large distances beween them. Above the monofilament curve the magnetization in the perpendicular configuration is shown, for distances between the filaments from $2 R$ to $0.1 R$, below the magnetization in the parallel configuration, likewise from $2 R$ to $0.1 R$.

The demagnetization effects are quite pronounced at small distances and it is seen that the area of magnetization loops at field variations below $2 B_{\mathrm{p}}$ will be greatly influenced by this effect. Large increases in hysteresis

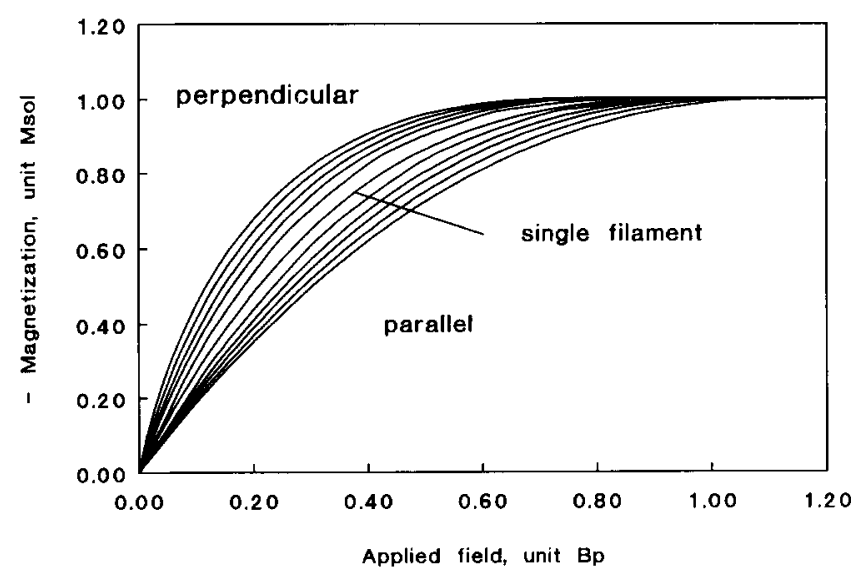

Figure 9 Magnetization of a row of nine filaments at distances $0.1 R, 0.3 R, 0.6 R, 1 R$ and $2 R$, perpendicular and parallel to the applied field, as a function of $\beta=B_{a} / B_{p}$. Magnetization of a monofilament is shown in the middle

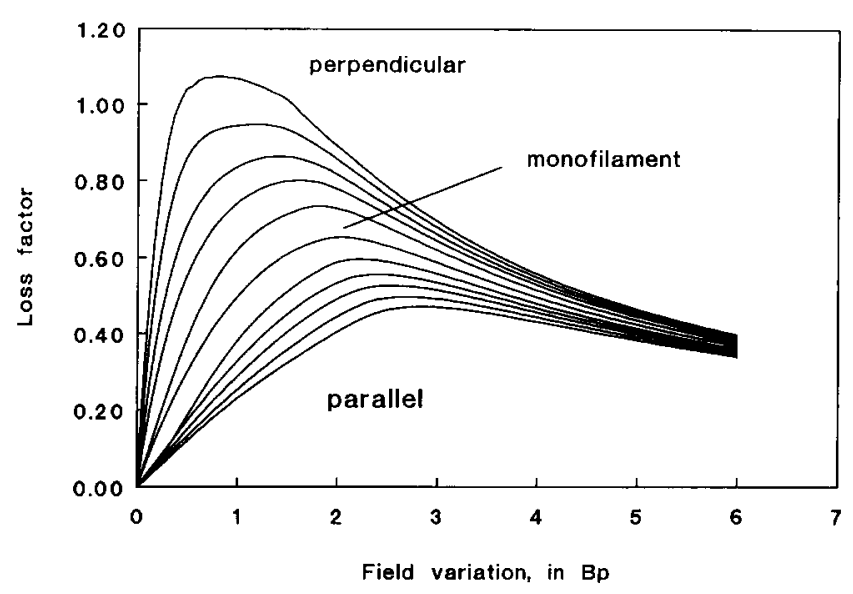

Figure 10 Normalized loss factor, $\Gamma(\delta)$, as a funciton of $\delta=\Delta B / B_{\mathrm{p}}$ for a row of nine filaments at distances $0.1 R, 0.3 R$, $0.6 R, 1 R$ and $2 R$, perpendicular and parallel to the applied field $\Gamma(\delta)$ for a monofilament is shown in the middle

losses when the field variation is smaller than $2 B_{\mathrm{p}}$ have been reported ${ }^{8}$ and have been explained as being due to demagnetization effects. In contrast to earlier theories, no assumptions of uniformity of the local field in the filament have to be made in the present model. Figure 10 shows the calculated loss factor, $\Gamma(\delta)$, for this configuration. It is seen that the losses below penetration can easily be different from losses in a monofilament by an order of magnitude. For field variations above $2 B_{\mathrm{p}}$ the effects decrease rapidly.

Superconducting wires usually contain a large number of filaments. When all the filaments are penetrated by the applied field, the magnetic moment of the assembly is smaller than that of a monofilament of the same area, as the magnetic moment is proportional to $R^{3}$. A filament in the interior region of the wire is surrounded on all sides by other filaments and the total field induced by neighbouring current distributions will generally be small. In cables or magnets wires are packed together. The influence of the induced current distributions in the filaments of one wire on the filaments in a neighbouring wire depends on the filament diameter and the general configuration. When the filament number is not large enough or when the effective filament diameter is large because of bridging, demagnetization effects may become noticeable. To compare the situation of a wire with the results previously shown for a row of nine monofilaments, calculations were performed for a wire which had 19 filaments with the same radius as the monofilaments. Five wires were stacked together in a row with a distance between them of $0.1 R_{\text {wire }}$, where $R_{\text {wire }}=6 R_{\text {fil }}$. The magnetization was calculated for this row in a configuration parallel and perpendicular to the applied field. From the magnetization the hysteresis losses can be determined.

Figure 11 shows the calculated loss factor, $\Gamma(\delta)$, for the single wire and both configurations, Although the demagnetization effects have decreased markedly in comparison to the results for the row of monofilaments with the same distance between them, the effect of neighbouring wires is still sizeable around $\Delta B=2 B_{\mathrm{p}}$. When the effective filament diameter is such that 


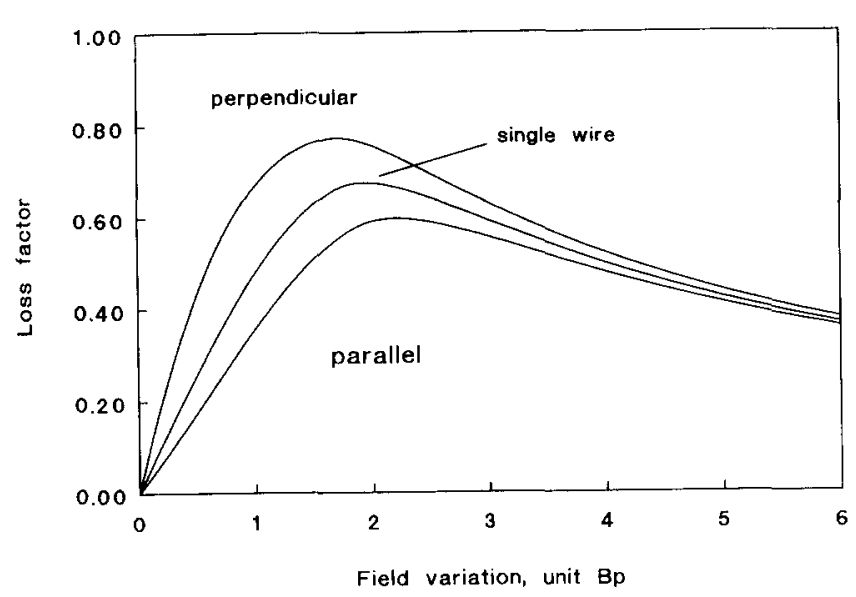

Figure 11 Normalized loss factor, $\Gamma(\delta)$, as a function of $\delta=\Delta B / B_{\mathrm{p}}$ for a row of five wires at a distance $0.1 R$, perpendicular and parallel to the applied field. $\Gamma(\delta)$ for a single wire is shown in the middle

$\Delta B \gg B_{\mathrm{p}}$ the demagnetization effects become negligible.

\section{Filament bridging}

In wires where the $\mathrm{Nb}_{3} \mathrm{Sn}$ filaments are designed to have very small diameters superconducting bridges may be formed between adjoining filaments during the heat treatment. Sharp increases in the hysteresis losses in certain wires have been reported by Goldfarb and Ekin ${ }^{9}$, who defined an effective diameter, $d_{\text {eff }}$, for the filaments which was several times larger than the designed filament diameter, $d_{\text {design. }}$. The measured magnetization of a wire in which the filaments are coupled by superconducting bridges is shown in Figure $7 b$. The bridged filaments behave as an extended superconductor with a peculiar shape. To investigate the behaviour of such a configuration, 37 solid circular filaments were connected by bridges, as shown in Figure 12. Without bridges the magnetization of the 37 filaments was calculated to be almost equal to that of a monofilament.

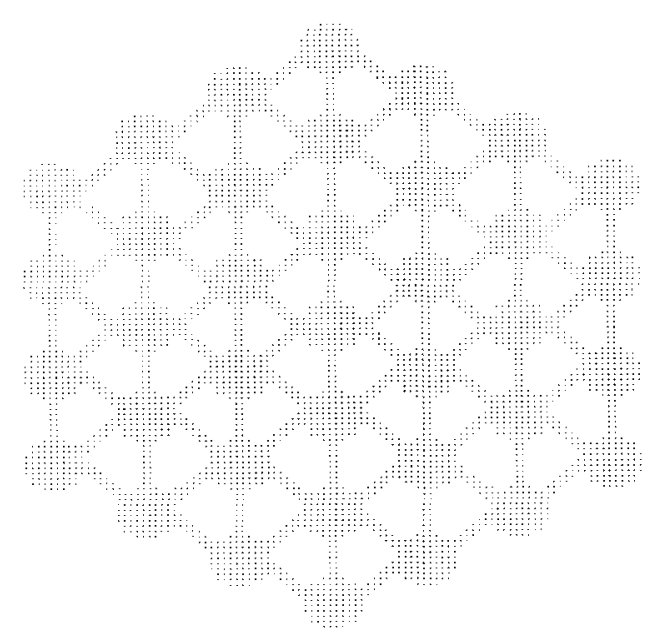

Figure 12 Configuration of 37 solid filaments coupled by superconducting bridges, every dot representing a grid cell

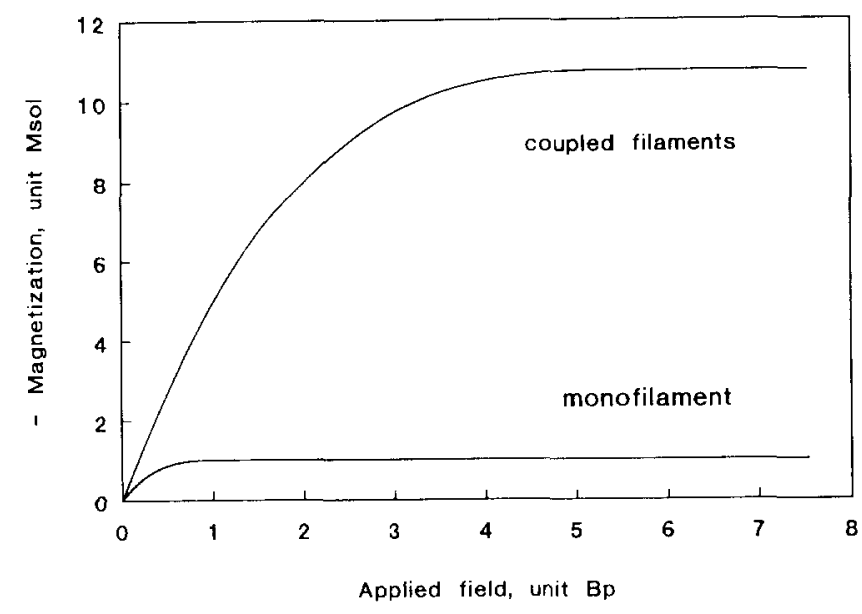

Figure 13 Magnetization of a configuration of 37 solid filaments coupled by superconducting bridges and magnetization of a monofilament for comparison

The magnetization for the case with bridges was calculated as a function of the applied field and is shown in Figure 13, $M$ and $B$ being scaled to the saturated magnetization, $M_{\text {sol }}$, and the penetration field, $B_{\mathrm{p}}$, of a monofilament. It is seen that the saturated magnetization for the bridged configuration, $f_{\mathrm{m}}=10.7$ times greater and the effective penetration field, $\mathrm{f}_{\mathrm{b}}=5.5$ times greater, relative to a monofilament. This means that the magnetization increases more relative to the penetration field, so there is no longer a fixed relation between the penetration field, $B_{\mathrm{p}}$, and the saturated magnetization, $M_{\text {sal }}$, as was the case in a solid filament [see Equation (6) ]. An observed increase in $B_{\mathrm{p}}$ does not mean that the magnetization has increased by the same amount. As hysteresis losses for $\Delta B \gg 2 B_{\mathrm{p}}$ are almost entirely determined by $M_{\mathrm{sat}}$ and not by $B_{\mathrm{p}}$, the increase in the effective penetration field is not a good indicator for the expected increase in the hysteresis losses. For $\Delta B \gg B_{\mathrm{p}}$ hysteresis losses, $Q_{\mathrm{h}}$, are proportional to $M_{\text {sat }} \Delta B$. When the hysteresis losses, $Q_{\text {exp }}$, measured experimentally for a case where $\Delta B \gg B_{\mathrm{p}}$ are compared with the calculated losses, $Q_{\text {cal }}$, which are based on the non-bridged filaments with the designed filament diameter, the ratio will be approximately equal to the factor $f_{\mathrm{m}}$. A meaningful definition for the effective diameter of the filaments would be $d_{\mathrm{eff}}=f_{\mathrm{m}} d_{\text {design }}$ or

$d_{\text {eff }}=\frac{Q_{\text {exp }}}{Q_{\text {cal }}} d_{\text {design }}$, where $\Delta B \gg B_{\text {p.eff }}$

For $\Delta B \gg B_{\mathrm{p} . \mathrm{eff}}$ the effective diameter thus defined can be used in the known expressions for the calculation of hysteresis losses. In reality bridging is more complicated than the relatively simple model of Figure 12 which has been used here. The model shows, however, that the relation between the penetration field and the saturated magnetization in a wire with bridged filaments is different from the relation found for the case of a wire with isolated solid filaments. When $\Delta B<B_{\mathrm{p} \text {,eff }}$ hysteresis losses can only be calculated when the rela- 
tion between the applied field and the resulting magnetization is known.

\section{Conclusions}

With the numerical model the current distribution in superconductors can be determined as a function of the applied field for superconductors of arbitrary shape, such as hollow filaments, multifilament systems or filaments which are connected by superconducting bridges. Knowing this, current distribution quantities such as hysteresis losses over a closed cycle of the external field and also the instantaneous heat dissipation can be calculated. Measured hysteresis losses in hollow filaments show good agreement with calculated losses. Measured instantaneous heat dissipation agrees qualitatively with calculated losses. An effective filament diameter for bridged filaments is defined which is based not on the observed increase of the penetration field but on the measured increase of the hysteresis losses.

\section{References}

1 Matsushita, T. Electromagnetic phenomena and hysteresis losses in superconductors Cryogenics (1989) 30 314-323

2 Pang, C.Y., Maclaren, P.G. and Campbell, A.M. Losses in NbTi multifilamentary composite when exposed to transverse alternating and rotating fields IEEE Trans Magn (1981) MAG-17(1) 134-137

3 Hartmann, R.A., Dijkstra, D., van Beckum, F.P.H. and van de Klundert, L.J.M. Calculations on the current density and the voltage-current relation under a.c. conditions of filaments Cryogenics (1989) $29101-108$

4 Eikelboom, J.A., Hartmann, R.A. and van de Klundert, L.J.M. Hysteresis losses in hollow filaments IEEE Trans Magn (1989) MAG25(2) $1968-1971$

5 Eikelboom, J.A., Hartmann, R.A. and van de Klundert, L.J.M. The power balance and losses in superconducting hollow filaments with transport currents Proc MT 11 Conf (1989) 1049-1054

6 Elen, J.D. and Franken, W.M.P. Niobium - tin wires by the ECN powder method Cryogenics (1987) 27 106-107

7 Rem, P.C. Numerical models for a.c. superconductors PhD Thesis University of Twente, The Netherlands (1986)

8 Zenkevitch, V.B., Zheltov, V.V. and Romanyuk, A.S. Hysteresis losses in superconductors of round cross-section with collective interaction Cryogenics (1978) 18 93-99

9 Goldfarb, R.B. and Ekin, J.W. Hysteresis losses in fine filament internal-tin superconductors Cryogenics (1986) $26478-481$ 